\title{
Strichartz Inequalities for the Wave Equation with the Full Laplacian on H-Type Groups
}

\author{
Heping Liu and Manli Song \\ School of Mathematical Sciences, Peking University, Beijing 100871, China \\ Correspondence should be addressed to Manli Song; songmanli@pku.edu.cn \\ Received 6 December 2013; Accepted 31 January 2014; Published 16 March 2014 \\ Academic Editor: Dashan Fan
}

Copyright (C) 2014 H. Liu and M. Song. This is an open access article distributed under the Creative Commons Attribution License, which permits unrestricted use, distribution, and reproduction in any medium, provided the original work is properly cited.

We generalize the dispersive estimates and Strichartz inequalities for the solution of the wave equation related to the full Laplacian on H-type groups, by means of Besov spaces defined by a Littlewood-Paley decomposition related to the spectral of the full Laplacian. The dimension of the center on those groups is $p$ and we assume that $p>1$. A key point consists in estimating the decay in time of the $L^{\infty}$ norm of the free solution. This requires a careful analysis due also to the nonhomogeneous nature of the full Laplacian.

\section{Introduction}

The aim of this paper is to study Strichartz inequalities for the solution for the following Cauchy problem of the wave equation related to the full Laplacian on H-type groups $G$ with topological dimension $n$ and homogeneous dimension $N$ :

$$
\begin{gathered}
\partial_{t t} u+\mathscr{L} u=f \in L^{1}\left((0, T), L^{2}\right), \\
\left.u\right|_{t=0}=u_{0} \in \dot{B}_{2,2}^{1}, \\
\left.\partial_{t} u\right|_{t=0}=u_{1} \in L^{2},
\end{gathered}
$$

where $\mathscr{L}$ is the full Laplacian on $G$ and the Besov spaces $\dot{B}_{q, r}^{\rho}(\mathscr{L})$ (written by $\dot{B}_{q, r}^{\rho}$ for short) are defined by a Littlewood-Paley decomposition related to the full Laplacian. In [1], Bahouri et al. found sharp dispersive estimates and Strichartz inequalities for the Cauchy problem for the wave equation related to the Kohn-Laplacian $\Delta$ on the Heisenberg group, using the Besov spaces $\dot{B}_{q, r}^{\rho}(\Delta)$. In [2], Furioli et al. studied the corresponding Cauchy problem for the wave equation with the full Laplacian on the Heisenberg group, using the Besov spaces $\dot{B}_{q, r}^{\rho}$. They also proved that there was no hope to obtain a dispersive inequality as in Theorem 1 with the space $\dot{B}_{q, r}^{\rho}(\Delta)$. Later, in [3], Del Hierro generalized the dispersive and Strichartz estimates for the wave equation on H-type groups, using the Besov spaces $\dot{B}_{q, r}^{\rho}(\Delta)$.

In this paper, we will show that the wave equation related to the full Laplacian on $\mathrm{H}$-type groups is also dispersive, using the Besov space $\dot{B}_{q, r}^{\rho}$. To deal with the problem, we have to pay attention to two points compared with $[2,3]$. On the one hand, the full Laplacian does not have the homogeneous properties. On the other hand, the dimension of the center of H-type groups is in general bigger than 1 (actually, in the $\mathrm{H}$-type groups, only the Heisenberg groups have a one dimensional centre).

It is well known that the general solution (1) can be written as $u=v+w$ where $v$ is a solution of (1) with $f=0$ and $w$ is the solution of (1) with $u_{0}=u_{1}=0$. They are classically given by

$$
\begin{aligned}
& v(t)=\cos (t \sqrt{\mathscr{L}}) u_{0}+\frac{\sin (t \sqrt{\mathscr{L}})}{\sqrt{\mathscr{L}}} u_{1}, \\
& w(t)=\int_{0}^{t} \frac{\sin ((t-\tau) \sqrt{\mathscr{L}})}{\sqrt{\mathscr{L}}} f(\tau) d \tau .
\end{aligned}
$$

We can now state the main results of the paper. As always when dealing with Strichartz inequalities, we prove first the following dispersive inequality on $v$. 
Theorem 1. Let $\rho \in[n-1 / 2, n+1 / 2]$ and $u_{0} \in \dot{B}_{1,1}^{\rho}, u_{1} \in \dot{B}_{1,1}^{\rho-1}$. Then there exists a constant $C>0$, which does not depend on $u_{0}, u_{1}$, such that

$$
\|v(t)\|_{L^{\infty}(G)} \leq C|t|^{-p / 2}\left(\left\|u_{0}\right\|_{\dot{B}_{1,1}^{\rho}}+\left\|u_{1}\right\|_{\dot{B}_{1,1}^{\rho-1}}\right), \quad t \in \mathbb{R}^{*} .
$$

The Strichartz inequalities we have obtained are listed as follows.

Theorem 2. Let $q_{1}, q_{2}, r_{1}, r_{2} \in[2, \infty]$ and $\rho_{1}, \rho_{2} \in \mathbb{R}$ such that

(a)

$$
\frac{2}{q_{i}}=p\left(\frac{1}{2}-\frac{1}{r_{i}}\right) ; \quad i=1,2
$$

(b)

$$
\begin{aligned}
& -\left(n+\frac{1}{2}\right)\left(\frac{1}{2}-\frac{1}{r_{1}}\right)+1 \\
& \quad \leq \rho_{1} \leq-\left(n-\frac{1}{2}\right)\left(\frac{1}{2}-\frac{1}{r_{1}}\right)+1,
\end{aligned}
$$

(c)

$$
-\left(n+\frac{1}{2}\right)\left(\frac{1}{2}-\frac{1}{r_{1}}\right) \leq \rho_{2} \leq-\left(n-\frac{1}{2}\right)\left(\frac{1}{2}-\frac{1}{r_{1}}\right),
$$

except for $\left(q_{i}, r_{i}, p\right)=(2, \infty, 2)$. Let $q_{i}^{\prime}$, $r_{i}^{\prime}$ denote the conjugate exponent of $q_{i}$ and $r_{i}$. Then the following estimates are satisfied:

$$
\begin{gathered}
\|v\|_{L^{q_{1}\left(\mathbb{R}, \dot{B}_{r_{1}, 2}^{\rho_{1}}\right)}}+\left\|\partial_{t} v\right\|_{L^{q_{1}}\left(\mathbb{R}, \dot{B}_{r_{1}, 2}^{\rho_{1}-1}\right)} \leq C\left(\left\|u_{0}\right\|_{\dot{B}_{2,2}^{1}}+\left\|u_{1}\right\|_{L^{2}}\right), \\
\|w\|_{L^{q_{1}}\left((0, T), \dot{B}_{r_{1}, 2}^{\rho_{1}}\right)}+\left\|\partial_{t} w\right\|_{L^{q_{1}\left((0, T), \dot{B}_{r_{1}, 2}^{\rho_{1}-1}\right.}} \leq C\|f\|_{L_{2}^{q_{2}^{\prime}\left((0, T), \dot{B}_{r_{2}^{\prime}, 2}^{-\rho_{2}}\right.}},
\end{gathered}
$$

where the constant $C>0$ does not depend on $u_{0}, u_{1}, f$ or $T$.

Thus, it is natural to wonder whether such a generalization for Strichartz inequalities, obtained for the wave equation on H-type groups (with full Laplacian), remains true also for the corresponding Schrödinger equation:

$$
\begin{gathered}
\partial_{t} u-i \mathscr{L} u=f \in L^{1}\left((0, T), L^{2}\right), \\
\left.u\right|_{t=0}=u_{0} \in \dot{B}_{2,2}^{1} .
\end{gathered}
$$

We shall address this problem in a forthcoming paper [4].

\section{H-Type Groups and Spherical Fourier Transform}

2.1. H-Type Groups. Let $\mathfrak{g}$ be a two-step nilpotent Lie algebra endowed with an inner product $\langle\cdot, \cdot\rangle$. Its center is denoted by $\mathfrak{z} \cdot \mathfrak{g}$ is said to be of $\mathrm{H}$-type if $\left[\mathfrak{z}^{\perp}, z^{\perp}\right]=z$ and for every $s \in \mathfrak{z}$, the map $J_{s}: z^{\perp} \rightarrow z^{\perp}$ defined by

$$
\left\langle J_{s} u, w\right\rangle:=\langle s,[u, w]\rangle, \quad \forall u, w \in \mathfrak{z}^{\perp}
$$

is an orthogonal map whenever $|s|=1$.
An H-type group is a connected and simply connected Lie group $G$ whose Lie algebra is of H-type.

For a given $0 \neq a \in z^{*}$, the dual of $z$, we can define a skewsymmetric mapping $B(a)$ on $z^{\perp}$ by

$$
\langle B(a) u, w\rangle=a([u, w]), \quad \forall u, w \in z^{\perp} .
$$

We denote by $z_{a}$ the element of $z$ determined by

$$
\langle B(a) u, w\rangle=a([u, w])=\left\langle J_{z_{a}} u, w\right\rangle .
$$

Since $B(a)$ is skew symmetric and nondegenerate, the dimension of $z^{\perp}$ is even; that is, $\operatorname{dim} z^{\perp}=2 d$.

For a given $0 \neq a \in z^{*}$, we can choose an orthonormal basis

$$
\left\{E_{1}(a), E_{2}(a), \ldots, E_{d}(a), \bar{E}_{1}(a), \bar{E}_{2}(a), \ldots, \bar{E}_{d}(a)\right\}
$$

of $z^{\perp}$ such that

$$
\begin{gathered}
B(a) E_{i}(a)=\left|z_{a}\right| J_{z_{a} /\left|z_{a}\right|} E_{i}(a)=|a| \bar{E}_{i}(a), \\
B(a) \bar{E}_{i}(a)=-|a| E_{i}(a) .
\end{gathered}
$$

We set $p=\operatorname{dim} z$. Throughout this paper we assume that $p>1$. We can choose an orthonormal basis $\left\{\epsilon_{1}, \epsilon_{2}, \ldots, \epsilon_{p}\right\}$ of $z$ such that $a\left(\epsilon_{1}\right)=|a|, a\left(\epsilon_{j}\right)=0, j=2,3, \ldots, p$. Then we can denote the element of $\mathfrak{g}$ by

$$
(z, t)=(x, y, t)=\sum_{i=1}^{d}\left(x_{i} E_{i}+y_{i} \bar{E}_{i}\right)+\sum_{j=1}^{p} s_{j} \epsilon_{j} .
$$

We identify $G$ with its Lie algebra $\mathfrak{g}$ by exponential map. The group law on $\mathrm{H}$-type group $\mathrm{G}$ has the form

$$
(z, s)\left(z^{\prime}, s^{\prime}\right)=\left(z+z^{\prime}, s+s^{\prime}+\frac{1}{2}\left[z, z^{\prime}\right]\right) \text {, }
$$

where $\left[z, z^{\prime}\right]_{j}=\left\langle z, U^{j} z^{\prime}\right\rangle$ for a suitable skew-symmetric matrix $U^{j}, j=1,2, \ldots, p$.

Theorem 3. $G$ is an H-type group with underlying manifold $\mathbb{R}^{2 d+p}$, with the group law (15), and the matrix $U^{j}, j=$ $1,2, \ldots, p$ satisfies the following conditions.

(i) $U^{j}$ is a $2 d \times 2 d$ skew-symmetric and orthogonal matrix, $j=1,2, \ldots, p$.

(ii) $U^{i} U^{j}+U^{j} U^{i}=0, i, j=1,2, \ldots, p$ with $i \neq j$.

Proof. See [5].

Remark 4. It is well know that H-type algebras are closely related to Clifford modules (see [6]). H-type algebras can be classified by the standard theory of Clifford algebras. Specially, on H-type group $G$, there is a relation between the dimension of the center and its orthogonal complement space. That is $p+1 \leq 2 d$ (see [7]). 
Remark 5 . We identify $G$ with $\mathbb{R}^{2 d} \times \mathbb{R}^{p}$. We shall denote the topological dimension of $G$ by $n=2 d+p$. Following Folland and Stein (see [8]), we will exploit the canonical homogeneous structure, given by the family of dilations $\left\{\delta_{r}\right\}_{r>0}$,

$$
\delta_{r}(z, s)=\left(r z, r^{2} s\right) .
$$

We then define the homogeneous dimension of $G$ by $N=$ $2 d+2 p$.

The left invariant vector fields which agree, respectively, with $\partial / \partial x_{j}, \partial / \partial y_{j}$ at the origin are given by

$$
\begin{aligned}
& X_{j}=\frac{\partial}{\partial x_{j}}+\frac{1}{2} \sum_{k=1}^{p}\left(\sum_{l=1}^{2 d} z_{l} U_{l, j}^{k}\right) \frac{\partial}{\partial s_{k}}, \\
& Y_{j}=\frac{\partial}{\partial y_{j}}+\frac{1}{2} \sum_{k=1}^{p}\left(\sum_{l=1}^{2 d} z_{l} U_{l, j+d}^{k}\right) \frac{\partial}{\partial s_{k}},
\end{aligned}
$$

where $z_{l}=x_{l}, z_{l+d}=y_{l}, l=1,2, \ldots, d$.

The vector fields $S_{k}=\partial / \partial s_{k}, k=1,2, \ldots, p$ correspond to the center of $G$. In terms of these vector fields we introduce the sub-Laplacian $\Delta$ and full Laplacian $\mathscr{L}$, respectively,

$$
\begin{aligned}
& \Delta=-\sum_{j=1}^{n}\left(X_{j}^{2}+Y_{j}^{2}\right)=-\Delta_{z}+\frac{1}{4}|z|^{2} \mathcal{S}-\sum_{k=1}^{p}\left\langle z, U^{k} \nabla_{z}\right\rangle S_{k} \\
& \mathscr{L}=\Delta+\mathcal{S},
\end{aligned}
$$

where

$$
\begin{gathered}
\Delta_{z}=\sum_{j=1}^{2 d} \frac{\partial^{2}}{\partial z_{j}^{2}}, \quad \mathcal{S}=-\sum_{k=1}^{p} \frac{\partial^{2}}{\partial s_{k}^{2}}, \\
\nabla_{z}=\left(\frac{\partial}{\partial z_{1}}, \frac{\partial}{\partial z_{2}}, \ldots, \frac{\partial}{\partial z_{2 d}}\right)^{t} .
\end{gathered}
$$

2.2. Spherical Fourier Transform. Korányi, Damek, and Ricci $($ see $[9,10])$ have computed the spherical functions associated to the Gelfand pair $(G, O(2 d)$ ) (we identify $O(2 d)$ with $\left.O(2 d) \otimes I d_{p}\right)$. They involve, as on the Heisenberg group, the Laguerre functions

$$
\mathbb{2}_{m}^{(\alpha)}(\tau)=L_{m}^{(\alpha)}(\tau) e^{-\tau / 2}, \quad \tau \in \mathbb{R}, m, \alpha \in \mathbb{N},
$$

where $L_{m}^{(\alpha)}$ is the Laguerre polynomial of type $\alpha$ and degree $m$.

We say a function $f$ on $G$ is radial if the value of $f(z, s)$ depends only on $|z|$ and $s$. We denote by $\mathcal{S}_{\mathrm{rad}}(G)$ and $L_{\mathrm{rad}}^{q}(G)$, $1 \leq q \leq \infty$ the spaces of radial functions in $\delta(G)$ and $L^{p}(G)$, respectively. In particular, the set of $L_{\text {rad }}^{1}(G)$ endowed with the convolution product

$$
f_{1} * f_{2}(g)=\int_{G} f_{1}\left(g g^{\prime-1}\right) f_{2}\left(g^{\prime}\right) d g^{\prime}, \quad g \in G
$$

is a commutative algebra.
Let $f \in L_{\text {rad }}^{1}(G)$. We define the spherical Fourier transform

$$
\begin{aligned}
\mathfrak{F}(f)(\lambda, m)= & \widehat{f}(\lambda, m)=\left(\begin{array}{c}
m+d-1 \\
m
\end{array}\right)^{-1} \\
& \times \int_{\mathbb{R}^{2 d+p}} e^{i \lambda s} f(z, s) \mathfrak{Q}_{m}^{(d-1)}\left(\frac{|\lambda|}{2}|z|^{2}\right) d z d s, \\
& m \in \mathbb{N}, \lambda \in \mathbb{R}^{p} .
\end{aligned}
$$

By a direct computation, we have $\mathfrak{F}\left(f_{1} * f_{2}\right)=\mathfrak{F}\left(f_{1}\right) \cdot \mathfrak{F}\left(f_{2}\right)$. Thanks to a partial integration on the sphere $S^{p-1}$ we deduce from the Plancherel theorem on the Heisenberg group its analogue for the H-type groups.

Proposition 6. For all $f \in \mathcal{S}_{\text {rad }}(G)$ such that

$$
\sum_{m \in \mathbb{N}}\left(\begin{array}{c}
m+d-1 \\
m
\end{array}\right) \int_{\mathbb{R}^{p}}|\widehat{f}(\lambda, m)||\lambda|^{d} d \lambda<\infty
$$

we have

$$
\begin{aligned}
f(z, s)= & \left(\frac{1}{2 \pi}\right)^{d+p} \sum_{m \in \mathbb{N}} \int_{\mathbb{R}^{p}} e^{-i \lambda s} \widehat{f}(\lambda, m) \mathfrak{Q}_{m}^{(d-1)} \\
& \times\left(\frac{|\lambda|}{2}|z|^{2}\right)|\lambda|^{d} d \lambda
\end{aligned}
$$

the sum being convergent in $L^{\infty}$ norm.

Moreover, if $f \in \mathcal{S}_{\text {rad }}(G)$, the functions $\mathscr{L} f$ are also in $\mathcal{S}_{\text {rad }}(G)$ and its spherical Fourier transform is given by

$$
\widehat{\mathscr{L} f}(\lambda, m)=\left((2 m+d)|\lambda|+|\lambda|^{2}\right) \widehat{f}(\lambda, m) .
$$

The full Laplacian $\mathscr{L}$ is a positive self-adjoint operator densely defined on $L^{2}(G)$. So by the spectral theorem, for any bounded Borel function $h$ on $\mathbb{R}$, we have

$$
\widehat{h(\mathscr{L}) f}(\lambda, m)=h\left((2 m+d)|\lambda|+|\lambda|^{2}\right) \widehat{f}(\lambda, m) .
$$

\section{Littlewood-Paley Decomposition}

In this paper we use the Besov spaces defined by a LittlewoodPaley decomposition related to the spectral of the full Laplacian $\mathscr{L}$. Let $R$ be a nonnegative, even function in $C_{0}^{\infty}(\mathbb{R})$ such that $\operatorname{supp} R \subseteq\{\tau \in \mathbb{R}: 1 / 2 \leq|\tau| \leq 4\}$ and

$$
\sum_{j \in \mathbb{Z}} R\left(2^{-2 j} \tau\right)=1, \quad \forall \tau \neq 0 .
$$

For $j \in \mathbb{Z}$, we denote by $\psi_{j}$ the kernel of the operator $R\left(2^{-2 j} \mathscr{L}\right)$ and we set $\Delta_{j} f=f * \psi_{j}$. As $R \in C_{0}^{\infty}(\mathbb{R})$, Hulanicki proved that $\psi_{j} \in \mathcal{S}_{\text {rad }}(G)$ (see [11]) and

$$
\widehat{\psi}_{j}(\lambda, m)=R\left(2^{-2 j}\left((2 m+d)|\lambda|+|\lambda|^{2}\right)\right) .
$$

By [12] (see Proposition 6), there exists $C>0$ such that

$$
\left\|\psi_{j}\right\|_{L^{1}(G)} \leq C, \quad \forall j \in \mathbb{Z} .
$$


By standard arguments (see [12], Proposition 9), we can deduce from (29) that

$$
\begin{gathered}
\left\|\mathscr{L}^{\sigma / 2} \Delta_{j} f\right\|_{L^{q}(G)} \leq C 2^{j \sigma}\left\|\Delta_{j} f\right\|_{L^{q}(G)}, \\
\sigma \in \mathbb{R}, j \in \mathbb{Z}, 1 \leq q \leq \infty, f \in \mathcal{S}^{\prime}(G),
\end{gathered}
$$

where both sides of (30) are allowed to be infinite.

By the spectral theorem, for any $f \in L^{2}(G)$, the following homogeneous Littlewood-Paley decomposition holds:

$$
f=\sum_{j \in \mathbb{Z}} \Delta_{j} f \quad \text { in } L^{2}(G) \text {. }
$$

So

$$
\|f\|_{L^{\infty}(G)} \leq \sum_{j \in \mathbb{Z}}\left\|\Delta_{j} f\right\|_{L^{\infty}(G)}, \quad f \in L^{2}(G)
$$

where both sides of (32) are allowed to be infinite.

Let $1 \leq q, r \leq \infty, \rho<N / q$. We define the homogeneous Besov space $\dot{B}_{q, r}^{\rho}$ as the set of distributions $f \in \mathcal{S}^{\prime}(G)$ such that

$$
\|f\|_{\dot{B}_{q, r}^{\rho}}=\left(\sum_{j \in \mathbb{Z}} 2^{j \rho r}\left\|\Delta_{j} f\right\|_{q}^{r}\right)^{1 / r}<\infty
$$

and $f=\sum_{j \in \mathbb{Z}} \Delta_{j} f$ in $\mathcal{S}^{\prime}(G)$.

We collect in the following proposition all the properties we need about the spaces $\dot{B}_{q, r}^{\rho}$.

Proposition 7. Let $q, r \in[1, \infty]$ and $\rho<N / q$.

(i) The space $\dot{B}_{q, r}^{\rho}$ is a Banach space with the norm $\|\cdot\|_{\dot{B}_{q, r}^{\rho}}$;

(ii) the definition of $\dot{B}_{q, r}^{\rho}$ does not depend on the choice of the function $R$ in the Littlewood-Paley decomposition;

(iii) for $-N / q^{\prime}<\rho<N / q$ the dual space of $\dot{B}_{q, r}^{\rho}$ is $\dot{B}_{q^{\prime}, r^{\prime}}^{-\rho}$;

(iv) for $\alpha \in[n, N]$ we have the continuous inclusion

$$
\dot{B}_{q_{1}, r}^{\rho_{1}} \subset \dot{B}_{q_{2}, r}^{\rho_{2}}, \quad \frac{1}{q_{1}}-\frac{\rho_{1}}{\alpha}=\frac{1}{q_{2}}-\frac{\rho_{2}}{\alpha}, \quad \rho_{1} \geq \rho_{2} ;
$$

(v) for all $q \in[2, \infty]$ we have the continuous inclusion $\dot{B}_{q, 2}^{0} \subset L^{q}$;

(vi) $\dot{B}_{2,2}^{0}=L^{2}$;

(vii) for $\theta \in[0,1]$ we have

$$
\left[\dot{B}_{q_{1}, r_{1}}^{\rho_{1}}, \dot{B}_{q_{2}, r_{2}}^{\rho_{2}}\right]_{\theta}=\dot{B}_{q, r}^{\rho}
$$

with $\rho=(1-\theta) \rho_{1}+\theta \rho_{2}, 1 / q=(1-\theta) / q_{1}+\theta / q_{2}$, and $1 / r=$ $(1-\theta) / r_{1}+\theta / r_{2}$.

We omit the proof of the proposition which is analogous to (see [2, Proposition 3.3]).

\section{Dispersive Estimates}

It is a very classical way to get a dispersive estimate if we want to reach Strichartz inequalities. Hence, first what we want to do is to get a dispersive estimate $\left\|e^{-i t \sqrt{\mathscr{L}}} \psi_{j}\right\|_{L^{\infty}(G)}$.

Our main tool is to apply oscillating integral estimates to the wave equation. First of all, we recall the stationary phase lemma (see [13, Chapter VIII]).

Lemma 8 (stationary phase estimate). Let $g \in C^{\infty}([a, b])$ be real valued such that

$$
\left|g^{\prime \prime}(x)\right| \geq \delta
$$

for any $x \in[a, b]$ with $\delta>0$. Then for any function $h \in$ $C^{\infty}([a, b])$, there exists a constant $C$ which does not depend on $\delta, a, b, g$ or $h$, such that

$$
\left|\int_{a}^{b} e^{i g(x)} h(x) d x\right| \leq C \delta^{-1 / 2}\left[\|h\|_{\infty}+\int_{a}^{b}\left|h^{\prime}(x)\right| d x\right] .
$$

Next, we will need some estimates of the Laguerre functions.

Lemma 9. Consider the following:

$$
\left|\left(\tau \frac{d}{d \tau}\right)^{\alpha} \mathbf{\Omega}_{m}^{(d-1)}(\tau)\right| \leq C_{\alpha, d}(2 m+d)^{d-1 / 4}
$$

for all $0 \leq \alpha \leq d$

Proof. We refer the reader to the proof of Lemma 3.2 in [3].

Remark 10. In fact, for $0 \leq \alpha \leq d-1$, we have a better estimate

$$
\left|\left(\tau \frac{d}{d \tau}\right)^{\alpha} \mathfrak{\Omega}_{m}^{(d-1)}(\tau)\right| \leq C_{\alpha, d}(2 m+d)^{d-1} .
$$

Furthermore, we will exploit the following estimates, which can be easily proved by comparing the sums with the corresponding integrals.

Lemma 11. Fix $\beta \in \mathbb{R}$. There exists $C_{\beta}>0$ such that for $A>0$ and $d \in \mathbb{Z}_{+}$, and we have

$$
\begin{aligned}
& \sum_{\substack{m \in \mathbb{N} \\
2 m+d \geq A}}(2 m+d)^{\beta} \leq C_{\beta} A^{\beta+1}, \quad \beta<-1, \\
& \sum_{\substack{m \in \mathbb{N} \\
2 m+d \leq A}}(2 m+d)^{\beta} \leq C_{\beta} A^{\beta+1}, \quad \beta>-1 .
\end{aligned}
$$

Finally, we introduce the following properties of the Bessel functions. Let $J_{\mu}$ be the Bessel function of order $\mu>$ $-1 / 2$,

$$
J_{\mu}(r)=\frac{(r / 2)^{\mu}}{\Gamma(\mu+1 / 2) \pi^{1 / 2}} \int_{-1}^{1} e^{i r t}\left(1-t^{2}\right)^{\mu-1 / 2} d t .
$$

By $m$-fold integration by parts we obtain the following. 
Lemma 12. For any $m \in \mathbb{N}$,

$$
J_{m+1 / 2}=r^{-1 / 2} \sum_{k=0}^{m}\left(a_{k}^{+} e^{i r}+a_{k}^{-} e^{-i r}\right) r^{-k},
$$

where $a_{k}^{ \pm}$are complex coefficients.

Lemma 13. For any $m \in \mathbb{N}$,

$$
J_{m}(r)=e^{i r}\left[\frac{a_{+}}{r^{1 / 2}}+\phi_{+}(r)\right]+e^{-i r}\left[\frac{a_{-}}{r^{1 / 2}}+\phi_{-}(r)\right],
$$

where $\phi_{ \pm} \in \mathcal{S}\left(\mathbb{R}_{+}\right)$are such that

$$
\forall r>0, \quad\left|\phi_{ \pm}(r)\right| \leq r^{-1 / 2}, \quad\left|\phi_{ \pm}^{\prime}(r)\right| \leq r^{-3 / 2} .
$$

Proof. See the proof of Lemma 3.4 in [3].

We can now prove the following.

Lemma 14. There exists a $C>0$, which depends only on $d$ and $p$, such that for any $\rho \in[n-1 / 2, n+1 / 2], j \in \mathbb{Z}$, and $t \in \mathbb{R}^{*}=\mathbb{R} \backslash\{0\}$ we have

$$
\left\|e^{-i t \sqrt{\mathscr{L}}} \psi_{j}\right\|_{L^{\infty}(G)} \leq C|t|^{-1 / 2} 2^{j \rho} .
$$

Proof. Fixing $t \in \mathbb{R}^{*}, j \in \mathbb{Z}$, and $(z, s) \in G$ and by the inversion Fourier formula, we have

$$
\begin{aligned}
e^{-i t \sqrt{\mathscr{L}}} \psi_{j}(z, s)= & \left(\frac{1}{2 \pi}\right)^{d+p} \sum_{m \in \mathbb{N}} \int_{\mathbb{R}^{p}} e^{-i \lambda s} e^{-i t \sqrt{(2 m+d)|\lambda|+|\lambda|^{2}}} \\
& \times R\left(2^{-2 j}\left((2 m+d)|\lambda|+|\lambda|^{2}\right)\right) \\
& \times \mathbf{2}_{m}^{(d-1)}\left(\frac{|\lambda|}{2}|z|^{2}\right)|\lambda|^{d} d \lambda \\
= & \left(\frac{1}{2 \pi}\right)^{d+p} \sum_{m \in \mathbb{N}} I_{m}
\end{aligned}
$$

where

$$
\begin{aligned}
I_{m}= & \int_{\mathbb{R}^{p}} e^{-i \lambda s} e^{-i t \sqrt{(2 m+d)|\lambda|+|\lambda|^{2}}} R\left(2^{-2 j}\left((2 m+d)|\lambda|+|\lambda|^{2}\right)\right) \\
& \times \mathbf{2}_{m}^{(d-1)}\left(\frac{|\lambda|}{2}|z|^{2}\right)|\lambda|^{d} d \lambda
\end{aligned}
$$

and our assertion simply read

$$
\sum_{m \in \mathbb{N}}\left|I_{m}\right| \lesssim \begin{cases}|t|^{-1 / 2} 2^{j(2 d+p-1 / 2)}, & j>0 \\ |t|^{-1 / 2} 2^{j(2 d+p+1 / 2)}, & j \leq 0\end{cases}
$$

Putting $\sigma=s / t$ and $M=2 m+d$, we first integrate on $\mathbb{R}^{+}$, and then

$$
\begin{aligned}
I_{m}= & \int_{\mathbb{R}^{p}} e^{-i t\left(\sigma \cdot \lambda+\sqrt{M|\lambda|+|\lambda|^{2}}\right)} R\left(2^{-2 j}\left(M|\lambda|+|\lambda|^{2}\right)\right) \\
& \times \mathbf{2}_{m}^{(d-1)}\left(\frac{|\lambda|}{2}|z|^{2}\right)|\lambda|^{d} d \lambda \\
= & \int_{S^{p-1}} I_{\epsilon, m} d \sigma(\epsilon)
\end{aligned}
$$

where

$$
\begin{aligned}
I_{\epsilon, m}= & \int_{0}^{+\infty} e^{-i t\left(\lambda \sigma \cdot \epsilon+\sqrt{M \lambda+\lambda^{2}}\right)} R\left(2^{-2 j}\left(M \lambda+\lambda^{2}\right)\right) \\
& \times \mathbf{\Omega}_{m}^{(d-1)}\left(\frac{\lambda}{2}|z|^{2}\right) \lambda^{d+p-1} d \lambda .
\end{aligned}
$$

Performing the change of variable $x=2^{-2 j} M \lambda$, we obtain

$$
I_{\epsilon, m}=2^{j(2 d+2 p)} K_{\epsilon, m},
$$

where

$$
K_{\epsilon, m}=\int_{0}^{+\infty} e^{-i t 2^{j} G_{j, \sigma, \epsilon, m}(x)} h_{j, z, m}(x) d x
$$

Here,

$$
\begin{aligned}
& G_{j, \sigma, \epsilon, m}(x)=\frac{2^{j}}{M}\left(x \sigma \cdot \epsilon+\sqrt{2^{-2 j} M^{2} x+x^{2}}\right), \\
& h_{j, z, m}(x)=R\left(x+\frac{2^{2 j}}{M^{2}} x^{2}\right) \mathfrak{\Omega}_{m}^{(d-1)}\left(\frac{2^{2 j-1} x|z|^{2}}{M}\right) \frac{x^{d+p-1}}{M^{d+p}} .
\end{aligned}
$$

So

$$
\operatorname{supp} h_{j, z, m} \subseteq\left\{x \in \mathbb{R}^{+}: \frac{1}{2} \leq x+\frac{2^{2 j}}{M^{2}} x^{2} \leq 4\right\}=\left[a_{j, m}, b_{j, m}\right],
$$

where

$$
a_{j, m}=\frac{1}{1+\sqrt{1+2^{2 j+1} M^{-2}}}, \quad b_{j, m}=\frac{8}{1+\sqrt{1+2^{2 j+4} M^{-2}}} .
$$

Note that

$$
a_{j, m}, b_{j, m} \sim \min \left(1,2^{-j} M\right) .
$$

For $x \in\left[a_{j, m}, b_{j, m}\right]$, we have

$$
G_{j, \sigma, \epsilon, m}^{\prime \prime}(x)=-\frac{2^{-3 j-2} M^{3}}{\left(2^{-2 j} M^{2} x+x^{2}\right)^{3 / 2}} .
$$

Because of (55), it is implied that

$$
2^{-2 j-1} M^{2} \leq 2^{-2 j} M^{2} x+x^{2} \leq 2^{-2 j+2} M^{2}, \quad x \in\left[a_{j, m}, b_{j, m}\right] .
$$

Therefore,

$$
2^{-5} \leq\left|G_{j, \sigma, \epsilon, m}^{\prime \prime}(x)\right| \leq 2^{-1 / 2}, \quad x \in\left[a_{j, m}, b_{j, m}\right]
$$

follows immediately from (58) and (59).

Moreover, by Lemma 9 and (57), one can easily verify that

$$
\begin{array}{r}
\left\|h_{j, z, m}\right\|_{L^{\infty}\left[a_{j, m}, b_{j, m}\right]}+\left\|h_{j, z, m}^{\prime}\right\|_{L^{1}\left[a_{j, m}, b_{j, m}\right]} \\
\lesssim \begin{cases}M^{-(p+1)}, & M \geq 2^{j}, \\
2^{-j(d+p-1)} M^{d-2}, & M<2^{j} .\end{cases}
\end{array}
$$


Applying the stationary phase Lemma 8, we obtain a consistent estimate

$$
\left|K_{\epsilon, m}\right| \lesssim \begin{cases}|t|^{-1 / 2} 2^{-j / 2} M^{-(p+1)}, & M \geq 2^{j}, \\ |t|^{-1 / 2} 2^{-j(d+p-1 / 2)} M^{d-2}, & M<2^{j}\end{cases}
$$

Hence, we have

$$
\left|I_{m}\right| \lesssim \begin{cases}|t|^{-1 / 2} 2^{j(2 d+2 p-1 / 2)} M^{-(p+1)}, & M \geq 2^{j} \\ |t|^{-1 / 2} 2^{j(d+p+1 / 2)} M^{d-2}, & M<2^{j}\end{cases}
$$

For $j \leq 0, \sum_{m \in \mathbb{N}}\left|I_{m}\right| \lesssim|t|^{-1 / 2} 2^{j(2 d+2 p-1 / 2)} \lesssim|t|^{-1 / 2} 2^{j(2 d+p+1 / 2)}$. For $j>0, \sum_{m \in \mathbb{N}}\left|I_{m}\right| \lesssim|t|^{-1 / 2} 2^{j(2 d+p-1 / 2)}$ follows from (63) by applying Lemma 11 separately to the sums $\sum_{M \geq 2^{j}}\left|I_{m}\right|$ and $\sum_{M<2^{j}}\left|I_{m}\right|$.

Next, we integrate first over $S^{p-1}$ to estimate $I_{m}$,

$$
\begin{aligned}
I_{m}= & \int_{0}^{+\infty} \widehat{d \sigma}(\lambda s) e^{-i t \sqrt{M \lambda+\lambda^{2}}} \\
& \times R\left(2^{-2 j}\left(M \lambda+\lambda^{2}\right)\right) \mathfrak{\Omega}_{m}^{(d-1)}\left(\frac{\lambda}{2}|z|^{2}\right) \lambda^{d+p-1} d \lambda,
\end{aligned}
$$

where

$$
\widehat{d \sigma}(\xi)=\int_{S^{p-1}} e^{-i x \cdot \xi} d \sigma(x)=2 \pi\left(\frac{|\xi|}{2 \pi}\right)^{(2-p) / 2} J_{(p-2) / 2}(|\xi|) .
$$

Case 1 ( $p$ is odd). Using Lemma 12, we put

$$
I_{m}=(2 \pi)^{p / 2} \sum_{ \pm} \sum_{k=0}^{(p-3) / 2} a_{k}^{ \pm} I_{m, k}^{ \pm}
$$

where

$$
\begin{aligned}
I_{m, k}^{ \pm}= & |s|^{(1-p) / 2-k} \int_{0}^{+\infty} e^{ \pm i \lambda|s|-i t \sqrt{M \lambda+\lambda^{2}}} \\
& \times R\left(2^{-2 j}\left(M \lambda+\lambda^{2}\right)\right) \mathfrak{\Omega}_{m}^{(d-1)}\left(\frac{\lambda}{2}|z|^{2}\right) \lambda^{d+(p-1) / 2-k} d \lambda .
\end{aligned}
$$

Analogous to what we have done in Lemma 14, we obtain

$$
\begin{aligned}
& \left|I_{m, k}^{ \pm}\right| \\
& \lesssim \begin{cases}|t|^{-1 / 2}|s|^{(1-p) / 2-k} 2^{j(2 d+p+1 / 2-2 k)} M^{-((p+3) / 2-k)}, & M \geq 2^{j}, \\
|t|^{-1 / 2}|s|^{(1-p) / 2-k} 2^{j(d+p / 2+1-k)} M^{d-2}, & M<2^{j} .\end{cases}
\end{aligned}
$$

Case 2 ( $p$ is even). Using Lemma 13, we put

$$
I_{m}=(2 \pi)^{p / 2} \sum_{ \pm} a_{ \pm}\left(I_{m, 0}^{ \pm}+\Upsilon_{m}^{ \pm}\right)
$$

where

$$
\begin{aligned}
\Upsilon_{m}^{ \pm}= & |s|^{(2-p) / 2} \int_{0}^{+\infty} e^{ \pm i \lambda|s|-i t \sqrt{M \lambda+\lambda^{2}}} \phi_{ \pm}(\lambda|s|) \\
& \times R\left(2^{-2 j}\left(M \lambda+\lambda^{2}\right)\right) \mathbf{\Omega}_{m}^{(d-1)}\left(\frac{\lambda}{2}|z|^{2}\right) \lambda^{d+p / 2} d \lambda
\end{aligned}
$$

and the estimate holds

$$
\begin{aligned}
& \left|Y_{m}^{ \pm}\right| \\
& \quad \lesssim \begin{cases}|t|^{-1 / 2}|s|^{(1-p) / 2} 2^{j(2 d+p+1 / 2)} M^{-(p+3) / 2}, & M \geq 2^{j}, \\
|t|^{-1 / 2}|s|^{(1-p) / 2} 2^{j(d+p / 2+1)} M^{d-2}, & M<2^{j} .\end{cases}
\end{aligned}
$$

To improve the time decay, we will try to apply $p$ times a noncritical phase estimate. First, we need to give an estimate of the derivatives of the phase function $G_{j, \sigma, \epsilon, m}$.

Lemma 15. For any $x \in\left[a_{j, m}, b_{j, m}\right], l \geq 2$, we obtain

$$
\left|G_{j, \sigma, \epsilon, m}^{(l)}(x)\right| \lesssim \begin{cases}1, & M \geq 2^{j}, \\ \left(2^{j} M^{-1}\right)^{l-2}, & M<2^{j} .\end{cases}
$$

Proof. According to (58), we have

$$
G_{j, \sigma, \epsilon, m}^{\prime \prime}(x)=-\frac{2^{-3 j-2} M^{3}}{(\varphi(x))^{3 / 2}},
$$

where

$$
\varphi(x)=2^{-2 j} M^{2} x+x^{2} .
$$

By a direct induction, for $l \geq 2$, we have

$$
\begin{aligned}
G_{j, \sigma, \epsilon, m}^{(l)}(x)= & \left(G_{j, \sigma, \epsilon, m}^{\prime \prime}\right)^{(l-2)}(x) \\
= & -2^{-3 j-2} M^{3} \\
& \times \sum_{l_{1}+2 l_{2}=l-2} C\left(l, l_{1}, l_{2}\right) \frac{\left(\varphi^{\prime}(x)\right)^{l_{1}}\left(\varphi^{\prime \prime}(x)\right)^{l_{2}}}{(\varphi(x))^{3 / 2+l-2-l_{2}}} .
\end{aligned}
$$

Because of

$$
\begin{gathered}
\varphi(x) \sim 2^{-2 j} M^{2}, \\
\varphi^{\prime}(x)=2^{-2 j} M^{2}+2 x, \\
\varphi^{\prime \prime}(x)=2,
\end{gathered}
$$

for any $x \in\left[a_{j, m}, b_{j, m}\right]$.

By (57), when $M \geq 2^{j}$, we have $x \sim 1$. Hence, (77) yields

$$
\varphi^{\prime}(x) \sim 2^{-2 j} M^{2} .
$$


Then, according to (75), (76), (78), and (79), we have

$$
\begin{aligned}
\left|G_{j, \sigma, \epsilon, m}^{(l)}(x)\right| & \leqslant 2^{-3 j-2} M^{3} \sum_{l_{1}+2 l_{2}=l-2}\left(2^{-2 j} M^{2}\right)^{-\left(3 / 2+l-2-l_{2}-l_{1}\right)} \\
& \leqslant 2^{-3 j-2} M^{3} \sum_{0 \leq l_{2} \leq[(l-2) / 2]}\left(2^{-2 j} M^{2}\right)^{-\left(3 / 2+l_{2}\right)} \\
& \leqslant 2^{-3 j-2} M^{3}\left(2^{-2 j} M^{2}\right)^{-3 / 2} \\
& \leq 1
\end{aligned}
$$

By (57), when $M \leq 2^{j}$, we have $x \sim 2^{-j} M$. Hence, (77) yields

$$
\varphi^{\prime}(x) \sim 2^{-j} M
$$

Similarly, we prove that

$$
\left|G_{j, \sigma, \epsilon, m}^{(l)}(x)\right| \lesssim\left(2^{j} M^{-1}\right)^{l-2} .
$$

Furthermore, we will exploit the following estimates for the derivatives of $h_{j, z, m}$.

Lemma 16. For any $x \in\left[a_{j, m}, b_{j, m}\right], 0 \leq l \leq d$, we have

$$
\left|h_{j, z, m}^{(l)}(x)\right| \lesssim \begin{cases}M^{-\left(p+\theta_{l}\right)}, & M \geq 2^{j}, \\ 2^{-j(d+p-l-1)} M^{d-l-\theta_{l}-1}, & M<2^{j},\end{cases}
$$

where

$$
\theta_{l}= \begin{cases}1, & 0 \leq l \leq d-1, \\ \frac{1}{4}, & l=d\end{cases}
$$

Proof. Recall that

$$
h_{j, z, m}(x)=R\left(x+\frac{2^{2 j}}{M^{2}} x^{2}\right) \mathfrak{\Omega}_{m}^{(d-1)}\left(\frac{2^{2 j-1} x|z|^{2}}{M^{2}}\right) \frac{x^{d+p-1}}{M^{d+p}} .
$$

By an induction we get

$$
\begin{aligned}
h_{j, z, m}^{(l)}(x)= & \sum_{\alpha \in \mathscr{F}} A(l, \alpha) R^{\left(\alpha_{1}\right)}\left(x+\frac{2^{2 j}}{M^{2}} x^{2}\right) \\
& \times\left(1+\frac{2^{2 j+1}}{M^{2}} x\right)^{\alpha_{2}}\left(\frac{2^{2 j+1}}{M^{2}}\right)^{\alpha_{3}} \\
& \times\left[\left(x \frac{d}{d x}\right)^{\alpha_{4}} \mathfrak{\Omega}_{m}^{(d-1)}\right]\left(\frac{2^{2 j-1} x|z|^{2}}{M^{2}}\right) \frac{x^{d+p-\alpha_{5}-1}}{M^{d+p}},
\end{aligned}
$$

where $\mathscr{F}=\left\{\alpha=\left(\alpha_{1}, \ldots, \alpha_{5}\right) \in \mathbb{N}^{5}: \alpha_{1}=\alpha_{2}+\alpha_{3}, \alpha_{1}+\alpha_{3}+\alpha_{5}=\right.$ $\left.l, \alpha_{4} \leq \alpha_{5}\right\}$.

Applying Lemma 9 and (57), Lemma 16 comes out easily.

We can now prove the following.
Lemma 17. There exists $a C>0$, which depends only ond and $p$, such that for any $\rho \in[n-1 / 2, n+1 / 2], j \in \mathbb{Z}$, and $t \in \mathbb{R}^{*}$ we have

$$
\left\|e^{-i t \sqrt{\mathscr{L}}} \psi_{j}\right\|_{L^{\infty}(G)} \leq C|t|^{-p / 2} 2^{j \rho} .
$$

Proof. From Lemma 14, it suffices to prove the case $|t|>1$. In the following, we only give a detailed proof about the case when $p$ is odd. For the case $p$ is even, the proof is similar. Recall that

$$
K_{\epsilon, m}=\int_{0}^{+\infty} e^{-i t 2^{j} G_{j, \sigma, \epsilon, m}(x)} h_{j, z, m}(x) d x
$$

where

$$
G_{j, \sigma, \epsilon, m}^{\prime}(x)=\frac{2^{j}}{M}\left(\sigma \cdot \epsilon+\sqrt{1+\frac{2^{-4 j-2} M^{4}}{2^{-2 j} M^{2} x+x^{2}}}\right) .
$$

For $j>0$, we divide $\mathbb{N}$ into three (possible empty) disjoint subsets:

$$
\begin{aligned}
& A_{1}=\left\{m \in \mathbb{N}: M \geq 2^{j},|\sigma| \lesssim 2^{-j} M\right\}, \\
& A_{2}=\left\{m \in \mathbb{N}: M \geq 2^{j},|\sigma| \gtrsim 2^{-j} M\right\}, \\
& A_{3}=\left\{m \in \mathbb{N}: M<2^{j}\right\} .
\end{aligned}
$$

Then our assertion reads

$$
\sum_{m \in A_{r}}\left|I_{m}\right| \lesssim|t|^{-p / 2} 2^{j(2 d+p-1 / 2)}, \quad r=1,2,3 .
$$

For $r=1$, by (89), we obtain

$$
\left|G_{j, \sigma, \epsilon, m}^{\prime}(x)\right| \geq 1, \quad \text { for any } x \in\left[a_{j, m}, b_{j, m}\right] .
$$

The phase function $G_{j, \sigma, \epsilon, m}^{\prime}(x)$ for $K_{\epsilon, m}$ has no critical points on $\left[a_{j, m}, b_{j, m}\right]$. By $Q$-fold integration by parts, we get

$$
K_{\epsilon, m}=\left(i t 2^{j}\right)^{-Q} \int_{0}^{+\infty} e^{-i t 2^{j} G_{j, \sigma, \epsilon, m}(x)} D^{Q} h_{j, z, m}(x) d x,
$$

where the differential operator $D$ is defined by

$$
D h_{j, z, m}(x)=\frac{d}{d x}\left(\frac{h_{j, z, m}(x)}{G_{j, \sigma, \epsilon, m}^{\prime}(x)}\right) .
$$

By a direct induction, we have

$$
\begin{aligned}
D^{Q} h_{j, z, m}= & \sum_{k=Q}^{2 Q} \sum_{\sum_{l=1}^{Q+1} l \alpha_{l}=k} C(\alpha, k, Q) \\
& \times \frac{h_{j, z, m}^{\left(\alpha_{1}\right)}\left(G_{j, \sigma, \epsilon, m}^{\prime \prime}\right)^{\alpha_{2}} \cdots\left(G_{j, \sigma, \epsilon, m}^{(Q+1)}\right)^{\alpha_{Q+1}}}{\left(G_{j, \sigma, \epsilon, m}^{\prime}\right)^{k}}
\end{aligned}
$$

with $\alpha=\left(\alpha_{1}, \alpha_{2}, \ldots, \alpha_{\mathrm{Q}+1}\right) \in\{0,1, \ldots, Q\} \times \mathbb{N}^{\mathrm{Q}}$. 
For any $l \geq 2$, Lemma 15 implies

$$
\left|G_{j, \sigma, \epsilon, m}^{(l)}(x)\right| \lesssim 1, \quad \text { for any } x \in\left[a_{j, m}, b_{j, m}\right] \text {. }
$$

The estimates (92) and (96) yield

$$
\left\|D^{Q} h_{j, z, m}\right\|_{\infty} \lesssim \sup _{0 \leq \alpha_{1} \leq Q}\left\|h_{j, z, m}^{\left(\alpha_{1}\right)}\right\|_{\infty} .
$$

Applying Lemma 16, we obtain

$$
\sup _{0 \leq \alpha_{1} \leq Q}\left\|h_{j, z, m}^{\left(\alpha_{1}\right)}\right\|_{\infty} \lesssim M^{-(p+1 / 4)}
$$

By (57),

$$
a_{j, m}, b_{j, m} \sim 1
$$

So

$$
\left|K_{\epsilon, m}\right| \lesssim|t|^{-Q_{2}-j Q} M^{-(p+1 / 4)} .
$$

It follows from (40) that

$$
\begin{aligned}
& \sum_{A_{1}}\left|I_{m}\right| \lesssim|t|^{-Q_{2}} 2^{j(2 d+2 p-Q)} \\
& \times \sum_{M \geq 2^{j}} M^{-(p+1 / 4)} \lesssim|t|^{-Q} 2^{j(2 d+p+3 / 4-Q)} .
\end{aligned}
$$

Let $Q=d$. Since $p \leq 2 d-1$ and $p>1$, we have $d>p / 2$ and $d \geq 2$. Hence,

$$
\sum_{A_{1}}\left|I_{m}\right| \lesssim|t|^{-d} 2^{j(d+p+3 / 4)} \leq|t|^{-p / 2} 2^{j(2 d+p-1 / 2)} .
$$

For $r=2$, the estimate (68) yields

$$
\begin{aligned}
\left|I_{m, k}^{ \pm}\right| & \lesssim|t|^{-p / 2-k} 2^{j(2 d+3 p / 2-k)} M^{-(p+1)} \\
& \lesssim|t|^{-p / 2} 2^{j(2 d+3 p / 2)} M^{-(p+1)} .
\end{aligned}
$$

Then it follows from (40) that

$$
\begin{aligned}
& \sum_{m \in A_{2}}\left|I_{m}\right| \lesssim|t|^{-p / 2} 2^{j(2 d+3 p / 2)} \\
& \times \sum_{M \geq 2^{j}} M^{-(p+1)} \lesssim|t|^{-p / 2} 2^{j(2 d+p / 2)} \lesssim|t|^{-p / 2} 2^{j(2 d+p-1 / 2)} .
\end{aligned}
$$

For $r=3$, when $|\sigma| \gtrsim 1$, the estimate (68) yields

$$
\begin{aligned}
\left|I_{m, k}^{ \pm}\right| & \lesssim|t|^{-p / 2-k} 2^{j(d+p / 2+1-k)} M^{d-2} \\
& \leq|t|^{-p / 2} 2^{j(d+p / 2+1)} M^{d-2} .
\end{aligned}
$$

Thanks to (41), we have

$$
\begin{aligned}
& \sum_{m \in A_{3}}\left|I_{m}\right| \lesssim|t|^{-p / 2} 2^{j(d+p / 2+1)} \\
& \quad \times \sum_{M<2^{j}} M^{d-2} \lesssim|t|^{-p / 2} 2^{j(2 d+p / 2)} \lesssim|t|^{-p / 2} 2^{j(2 d+p-1 / 2)} .
\end{aligned}
$$

When $|\sigma| \lesssim 1$, similar to $r=1$, the estimates

$$
\begin{aligned}
& \left|G_{j, \sigma, \epsilon, m}^{\prime}(x)\right| \gtrsim 2^{j} M^{-1}, \\
& \left|G_{j, \sigma, \epsilon, m}^{(l)}(x)\right| \lesssim\left(2^{j} M^{-1}\right)^{l-2}, \quad l \geq 2
\end{aligned}
$$

hold for any $x \in\left[a_{j, m}, b_{j, m}\right]$. Therefore,

$$
\begin{aligned}
\left\|D^{Q} h_{j, z, m}\right\|_{\infty} \lesssim & \sup _{0 \leq \alpha_{1} \leq Q}\left\|h_{j, z, m}^{\left(\alpha_{1}\right)}\right\|_{\infty} \\
& \times \sup _{\mathrm{Q} \leq k \leq 2 \mathrm{Q}} \sup _{\sum_{l=1}^{\mathrm{Q}+1} l \alpha_{l}=k}\left(2^{j} M^{-1}\right)^{\sum_{l=2}^{Q+1}(l-2) \alpha_{l}-k} .
\end{aligned}
$$

Because of

$$
\begin{aligned}
\sum_{l=2}^{\mathrm{Q}+1}(l-2) \alpha_{l}-k & =-\sum_{l=2}^{\mathrm{Q}+1} 2 \alpha_{l}-\alpha_{1} \\
& \leq \frac{-2}{(Q+1)} \sum_{l=1}^{\mathrm{Q}+1} l \alpha_{l}=-\frac{2 k}{(\mathrm{Q}+1)} \leq-\frac{2 Q}{(\mathrm{Q}+1)}
\end{aligned}
$$

and according to Lemma 16

$$
\sup _{0 \leq \alpha_{1} \leq Q}\left\|h_{j, z, m}^{\left(\alpha_{1}\right)}\right\|_{\infty} \lesssim 2^{-j(p+d-Q-1)} M^{d-Q-5 / 4}
$$

it follows that

$$
\left\|D^{\mathrm{Q}} h_{j, z, m}\right\|_{\infty} \leqslant 2^{-j(p+d+2 \mathrm{Q} /(\mathrm{Q}+1)-\mathrm{Q}-1)} M^{d+2 \mathrm{Q} /(\mathrm{Q}+1)-\mathrm{Q}-5 / 4} .
$$

Moreover, by (57),

$$
a_{j, m}, b_{j, m} \sim 2^{-j} M .
$$

Therefore, we obtain

$$
\begin{aligned}
\left|K_{\epsilon, m}\right| & \leqslant|t|^{-Q_{2}-j Q}\left\|D^{Q} h_{j, z, m}\right\|_{\infty} 2^{-j} M \\
& =|t|^{-Q} 2^{-j(p+d+2 Q /(Q+1))} M^{d+2 Q /(Q+1)-Q-1 / 4} .
\end{aligned}
$$

Let $Q=d$, and then

$$
\left|K_{\epsilon, m}\right| \lesssim|t|^{-d} 2^{-j(d+p+2 d /(d+1))} M^{2 d /(d+1)-1 / 4} .
$$

Because of (41) and $d>p / 2$,

$$
\begin{aligned}
& \sum_{A_{3}}\left|K_{\epsilon, m}\right| \lesssim|t|^{-p / 2} 2^{-j(d+p+2 d /(d+1))} \\
& \quad \times \sum_{M<2^{j}} M^{2 d /(d+1)-1 / 4} \lesssim|t|^{-p / 2} 2^{-j(d+p-3 / 4)} .
\end{aligned}
$$

Noticing that $d \geq 2$, we have

$$
\begin{aligned}
\sum_{A_{3}}\left|I_{m}\right| & \lesssim 2^{j(2 d+2 p)} \sum_{A_{3}}\left|K_{\epsilon, m}\right| \\
& \lesssim|t|^{-p / 2} 2^{j(d+p+3 / 4)} \leq|t|^{-p / 2} 2^{j(2 d+p-1 / 2)} .
\end{aligned}
$$


For $j \leq 0$, we divide $\mathbb{N}$ into two (possible empty) disjoint subsets

$$
\begin{aligned}
& B_{1}=\left\{m \in \mathbb{N}:|\sigma| \lesssim 2^{-j} M\right\}, \\
& B_{2}=\left\{m \in \mathbb{N}:|\sigma| \gtrsim 2^{-j} M\right\} .
\end{aligned}
$$

Then our assertion reads

$$
\sum_{m \in B_{r}}\left|I_{m}\right| \lesssim|t|^{-p / 2} 2^{j(2 d+p+1 / 2)}, \quad r=1,2 .
$$

For $B_{1}$, analogous to the case $A_{1}$ for $j>0$, we get

$$
\left|K_{\epsilon, m}\right| \lesssim|t|^{-Q_{2}-j Q} M^{-(p+1 / 4)} .
$$

So

$$
\begin{aligned}
& \sum_{m \in B_{1}}\left|I_{m}\right| \lesssim|t|^{-Q_{2}} 2^{j(2 d+2 p-Q)} \\
& \quad \times \sum_{m \in \mathbb{N}} M^{-(p+1 / 4)} \lesssim|t|^{-Q_{2}} 2^{j(2 d+2 p-Q)} .
\end{aligned}
$$

Let $Q=(p+1) / 2 \leq d$. Because of $p>1$, it is implied that

$$
\sum_{m \in B_{1}}\left|I_{m}\right| \lesssim|t|^{-p / 2} 2^{j(2 d+3 p / 2-1 / 2)} \lesssim|t|^{-p / 2} 2^{j(2 d+p+1 / 2)} .
$$

For $B_{2}$, the estimate (68) yields

$$
\begin{aligned}
\left|I_{m, k}^{ \pm}\right| & \lesssim|t|^{-p / 2-k} 2^{j(2 d+3 p / 2-k)} M^{-(p+1)} \\
& \lesssim|t|^{-p / 2} 2^{j(2 d+p+3 / 2)} M^{-(p+1)} .
\end{aligned}
$$

It follows that

$$
\begin{aligned}
\sum_{m \in B_{2}}\left|I_{m}\right| & \lesssim|t|^{-p / 2} 2^{j(2 d+p+3 / 2)} \sum_{m \in \mathbb{N}} M^{-(p+1)} \\
& \leqslant|t|^{-p / 2} 2^{j(2 d+p+3 / 2)} \lesssim|t|^{-p / 2} 2^{j(2 d+p+1 / 2)} .
\end{aligned}
$$

From Lemma 17, it is easy to obtain our sharp dispersive inequality.

Corollary 18. There exists $C>0$, which depends only on $d$ and $p$, such that for any $\rho \in[n-1 / 2, n+1 / 2], t \in \mathbb{R}^{*}$ and $f \in \mathcal{S}(G)$ we have

$$
\begin{aligned}
& \left\|e^{-i t \sqrt{\mathscr{L}}} f\right\|_{L^{\infty}(G)} \leq C|t|^{-p / 2}\|f\|_{\dot{B}_{1,1}^{\rho}}, \\
& \left\|e^{-i t \sqrt{\mathscr{L}}} f\right\|_{\dot{B}_{\infty, 1}^{-1}} \leq C|t|^{-p / 2}\|f\|_{\dot{B}_{1,1}^{\rho-1}} .
\end{aligned}
$$

We can obtain Corollary 18 by the same proof as in $[14$, Corollary 10].

The dispersive inequality in Theorem 1 is straightforward (see [2, Proposition 1.1]).

In the end of the section, let us show as in [3] the sharpness of the time decay in Corollary 18. First we recall the asymptotic expansion of oscillating integrals.
Proposition 19. Suppose $\phi$ is a smooth function on $\mathbb{R}^{p}$ and has a nondegenerate critical point at $x_{0}$. If $\psi$ is supported in a sufficiently small neighborhood of $x_{0}$, then

$$
\left|\int_{\mathbb{R}^{p}} e^{i t \phi(x)} \psi(x) d x\right| \sim|t|^{-p / 2}, \quad \text { as } t \longrightarrow \infty .
$$

A proof can be found in [13, Proposition 6, page 344].

Let $Q \in C_{0}^{\infty}\left(D_{0}\right)$ with $Q(d)=1$, where $D_{0}$ is a small neighborhood of $d$ such that $0 \notin D_{0}$. Then

$$
\widehat{u}_{0}(\lambda, m)=Q(|\lambda|) \delta_{m, 0}
$$

and $u_{1}:=0$ determines a solution of the Cauchy problem (1) with $f=0$ :

$$
\begin{aligned}
& u((z, s), t)= \cos (t \sqrt{\mathscr{L}}) u_{0} \\
&=C \int_{\mathbb{R}^{p}} e^{-i \lambda \cdot s-|\lambda||z|^{2} / 4} \cos \left(t \sqrt{d|\lambda|+|\lambda|^{2}}\right) \\
& \quad \times Q(|\lambda|)|\lambda|^{d} d \lambda .
\end{aligned}
$$

Consider $u\left(\left(0, t s_{0}\right), t\right)$ for a fixed $s_{0}$ such that $\left|s_{0}\right|=(3 / 2 \sqrt{2})$. This oscillating integral has a phase $\phi_{ \pm}(\lambda):=-\lambda \cdot s_{0} \pm$ $\sqrt{d|\lambda|+|\lambda|^{2}}$ with a unique critical point $\lambda_{0}^{ \pm}=\mp(2 \sqrt{2} d / 3) s_{0}$ which is not degenerate. Indeed, the Hessian is equal to

$$
\begin{aligned}
H(\lambda)=\mp & \left\{\frac{4|\lambda|^{2}+6 d|\lambda|+3 d^{2}}{4|\lambda|^{2}\left(d|\lambda|+|\lambda|^{2}\right)^{3 / 2}} \lambda_{k} \lambda_{l}\right. \\
& \left.-\delta_{k, l} \frac{d+2|\lambda|}{2|\lambda|\left(d|\lambda|+|\lambda|^{2}\right)^{1 / 2}}\right\}_{1 \leq k, l \leq p} .
\end{aligned}
$$

Let $s_{0}=(3 / 2 \sqrt{2})(0, \ldots, 0,1)$, so $\lambda_{0}^{ \pm}=\mp(2 \sqrt{2} d / 3) s_{0}=\mp(0$, $\ldots, 0, d)$. The Hessian at $\lambda_{0}^{ \pm}$is

$$
H\left(\lambda_{0}^{ \pm}\right)= \pm \frac{1}{8 \sqrt{2} d}\left\{\begin{array}{cccc}
12 & & & \\
& \ddots & & \\
& & 12 & \\
& & & -1
\end{array}\right\} \text {. }
$$

Applying asymptotic expansion of oscillating integrals, we get

$$
u\left(\left(0, t s_{0}\right), t\right) \sim|t|^{-p / 2} .
$$

\section{Strichartz Estimates}

We are now to prove our Strichartz estimates.

Proposition 20. For $i=1,2$, let $q_{i}, r_{i} \in[2, \infty]$ and $\rho_{i} \in \mathbb{R}$ such that

(a)

$$
\frac{2}{q_{i}}=p\left(\frac{1}{2}-\frac{1}{r_{i}}\right)
$$


(b)

$$
-\left(n+\frac{1}{2}\right)\left(\frac{1}{2}-\frac{1}{r_{i}}\right) \leq \rho_{i} \leq-\left(n-\frac{1}{2}\right)\left(\frac{1}{2}-\frac{1}{r_{i}}\right),
$$

except for $\left(q_{i}, r_{i}, p\right)=(2, \infty, 2)$. Then the following estimates are satisfied:

$$
\begin{gathered}
\left\|e^{-i t \sqrt{\mathscr{L}}} u_{0}\right\|_{L^{q_{1}}\left(\mathbb{R}, \dot{B}_{r_{1}, 2}^{\left.\rho_{1}\right)}\right.} \leq C\left\|u_{0}\right\|_{L^{2},} \\
\left\|\int_{0}^{t} e^{-i(t-\tau) \sqrt{\mathscr{L}}} f(\tau) d \tau\right\|_{L^{q_{1}\left((0, T), \dot{B}_{r_{1}, 2}^{\rho_{1}}\right)}} \leq C\|f\|_{L^{q_{2}^{\prime}\left((0, T), \dot{B}_{r_{2}^{\prime}, 2}^{-\rho_{2}}\right.}},
\end{gathered}
$$

where the constant $C>0$ does not depend on $u_{0}, f$, or $T$.

Once we have obtained the estimate in Lemma 17, the proof is classical and a good reference is, for example, the papers by Ginibre and Velo [15] or by Keel and Tao [16]. A detailed presentation in this framework is also given by [14] in the proof of Theorem 11.

Theorem 2 follows easily from the above proposition by the same proof that in [2].

In particular, by Besov interpolation we get the Strichartz estimates on Lebesgue spaces.

Theorem 21. Let $u$ be the solution of the Cauchy problem (1). If $q$ and $r$ satisfy $0 \leq 2 / q \leq p(1 / 2-1 / r)$ and $p[n(1 / 2-1 / r)-$ $1] \leq 1 / q \leq(p /(2 p-1))[N(1 / 2-1 / r)-1]$, then there exists a constant $C>0$, which does not depend on $u_{0}, u_{1}, f$, or $T$, such that the following estimate is satisfied:

$$
\|u\|_{L^{q}\left((0, T), L^{r}\right)} \leq C\left(\left\|u_{0}\right\|_{\dot{B}_{2,2}^{1}}+\left\|u_{1}\right\|_{L^{2}}+\|f\|_{L^{1}\left((0, T), L^{2}\right)}\right) .
$$

\section{Conflict of Interests}

The authors declare that there is no conflict of interests regarding the publication of this paper.

\section{Acknowledgments}

This work is performed while Manli Song studies as a joint Ph.D. student in the Mathematics Department of ChristianAlbrechts-Universität zu Kiel. Manli Song thanks Professor Detlef Müller for the hospitality of his Department. Thanks are also due to him for generous discussions and his continuous encouragement. Heping Liu is supported by National Natural Science Foundation of China under Grant no. 11371036 and the Specialized Research Fund for the Doctoral Program of Higher Education of China under Grant no. 2012000110059. Manli Song is supported by the China Scholarship Council under Grant no. 201206010098.

\section{References}

[1] H. Bahouri, P. Gérard, and C.-J. Xu, "Espaces de Besov et estimations de Strichartz généralisées sur le groupe de Heisenberg," Journal d'Analyse Mathématique, vol. 82, pp. 93-118, 2000.
[2] G. Furioli, C. Melzi, and A. Veneruso, "Strichartz inequalities for the wave equation with the full Laplacian on the Heisenberg group," Canadian Journal of Mathematics, vol. 59, no. 6, pp. 1301-1322, 2007.

[3] M. del Hierro, "Dispersive and Strichartz estimates on H-type groups," Studia Mathematica, vol. 169, no. 1, pp. 1-20, 2005.

[4] H. Liu and M. Song, "Strichartz inequalities for the Schrödinger equation with the full Laplacian on H-type groups," http://arxiv.org/abs/1402.4311.

[5] A. Bonfiglioli and F. Uguzzoni, "Nonlinear Liouville theorems for some critical problems on H-type groups," Journal of Functional Analysis, vol. 207, no. 1, pp. 161-215, 2004.

[6] H. M. Reimann, "H-type groups and Clifford modules," Advances in Applied Clifford Algebras, vol. 11, no. 2, supplement, pp. 277-287, 2001.

[7] A. Kaplan and F. Ricci, "Harmonic analysis on groups of Heisenberg type," in Harmonic Analysis, vol. 992 of Lecture Notes in Mathematics, pp. 416-435, Springer, Berlin, Germany, 1983.

[8] G. B. Folland and E. M. Stein, Hardy spaces on homogeneous groups, vol. 28 of Mathematical Notes, Princeton University Press, Princeton, NJ, USA, 1982.

[9] E. Damek and F. Ricci, "Harmonic analysis on solvable extensions of H-type groups," The Journal of Geometric Analysis, vol. 2, no. 3, pp. 213-248, 1992.

[10] A. Korányi, "Some applications of Gelfand pairs in classical analysis," in Harmonic Analysis and Group Representations, pp. 333-348, Liguori, Naples, Italy, 1982.

[11] A. Hulanicki, "A functional calculus for Rockland operators on nilpotent Lie groups," Studia Mathematica, vol. 78, no. 3, pp. 253-266, 1984.

[12] G. Furioli, C. Melzi, and A. Veneruso, "Littlewood-Paley decompositions and Besov spaces on Lie groups of polynomial growth," Mathematische Nachrichten, vol. 279, no. 9-10, pp. 1028-1040, 2006.

[13] E. M. Stein, Harmonic Analysis: Real-Variable Methods, Orthogonality and Oscillatory Integrals, Princeton University Press, Princeton, NJ, USA, 1993.

[14] G. Furioli and A. Veneruso, "Strichartz inequalities for the Schrödinger equation with the full Laplacian on the Heisenberg group," Studia Mathematica, vol. 160, no. 2, pp. 157-178, 2004.

[15] J. Ginibre and G. Velo, "Generalized Strichartz inequalities for the wave equation," Journal of Functional Analysis, vol. 133, no. 1, pp. 50-68, 1995.

[16] M. Keel and T. Tao, "Endpoint Strichartz estimates," The American Journal of Mathematics, vol. 120, no. 5, pp. 955-980, 1998. 


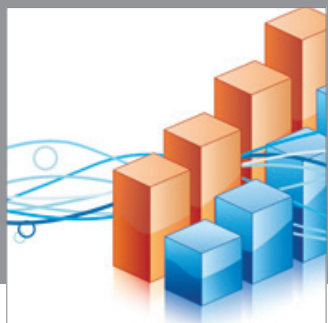

Advances in

Operations Research

mansans

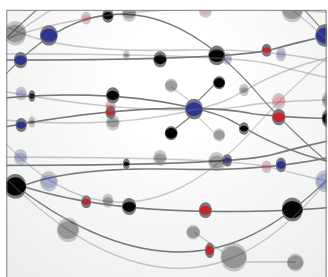

The Scientific World Journal
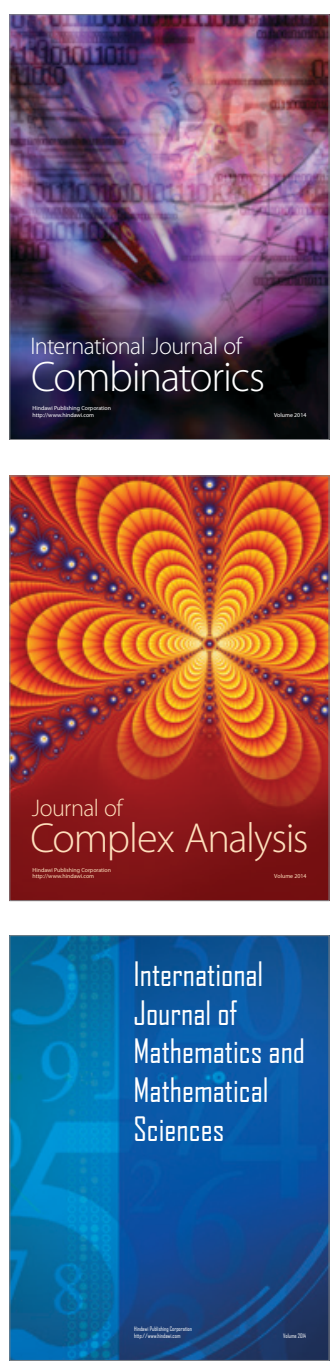
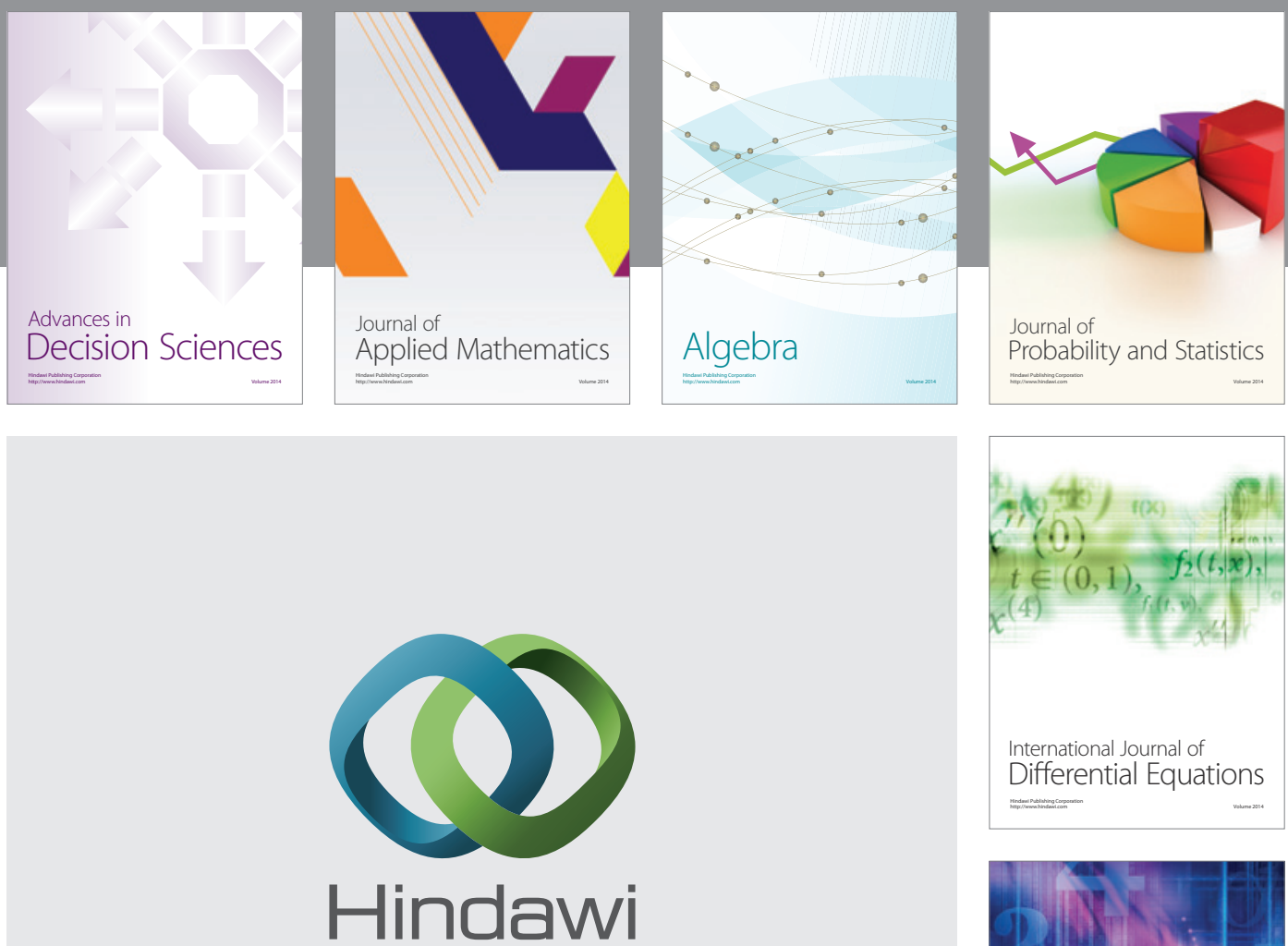

Submit your manuscripts at http://www.hindawi.com
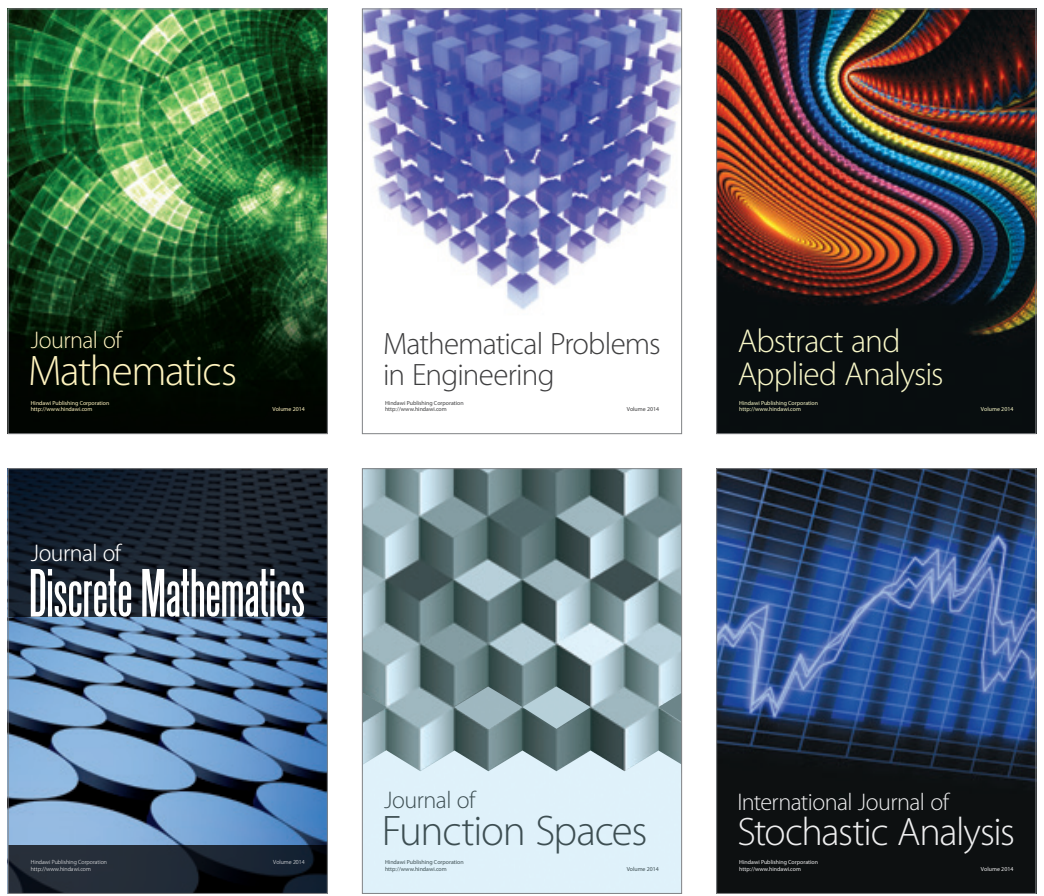

Journal of

Function Spaces

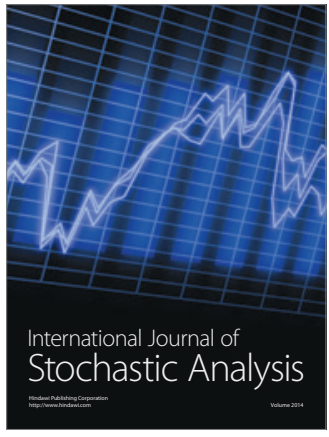

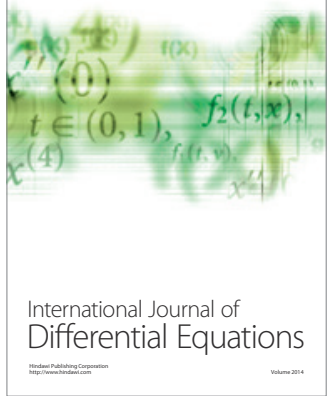
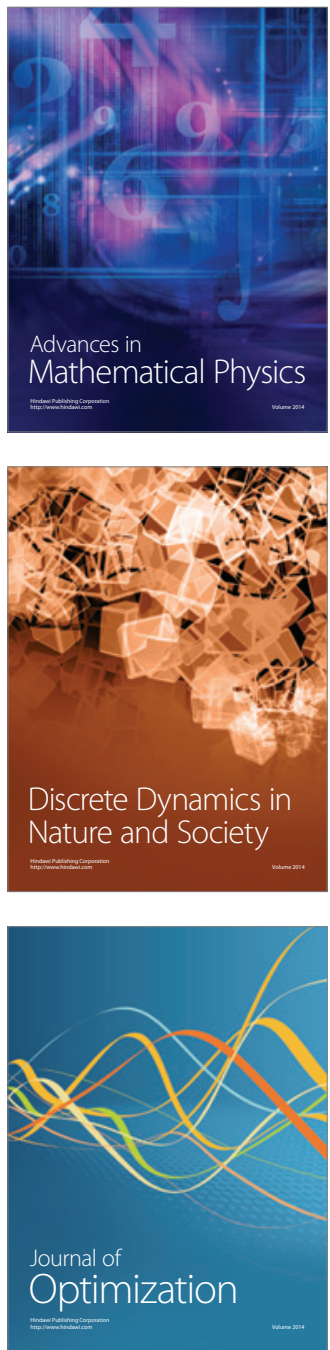